\title{
Co-evaluating emergency signage in coastal communities in Chile and Aotearoa: A case study
}

\author{
Keywords \\ Co Design; Co Evaluate; Information Design; Evacuation routes; Local knowledge.
}

Changes have significantly impacted the Design profession and disciplines during the last two decades, propelled by wicked problems confronting our societies. Climate change, loss of biodiversity, depletion of natural resources and the widening gap between rich and poor are just a few of the complex problems that require new approaches to problem-solving. In this uncertainty scenario, there is an increasing recognition that design, and designers can contribute to practical solutions. Many of these challenges are located outside the context of the business and consumer marketplace, and they require approaches that draw on multiple design specialisms and diverse worldviews. New design areas have been emerging to respond to this complexity, including Design for Social Innovation, Integrated Design and Transition Design (Irwin, 2015). This project heralds to contribute to discourses on how Design Practices can engage and contribute to problems in the field of emergencies, focused on understanding the issues and needs of coastal communities in Chile and Aotearoa, New Zealand. It is structured around an international collaboration between researchers and students from both countries. Generally, in the field of information design, the end-user, the ordinary citizen, is subjected to tests and testing in the final stages of design. We think that co-design methodologies seek to integrate future users early, considering them experts in the knowledge of their physical and social territory, such as a neighbourhood (Wilkomirsky, 2019). According to Petersen, Buscher, Kuhnert,
Schneider and Pottebaum (2015), co-design methods are "particularly valuable for eliciting ethical, legal, and social issues that would otherwise go unconsidered" (p.1). But by starting with visual systems already designed we thought that co-evaluation is a necessary first step that would allow us to grasp design judgment elements, among others: legibility, understanding of the message, clarity, and cross this information with the experience of the territory and its people for co-design improvements to what was projected in an abstract scale. Cockbill, May, and Mitchell, V. (2019) define co-design as "the act of designers, end-users, and other actors combining their views, skills, and perspectives at various stages of the design process in ways that influence the outcome" (p.568). In this first phase of this project, we compared visual information for evacuation routes including the administrative structure of the information and the visual display in different platforms in two cities: Whitianga and Puerto Montt, selected due the students local knowledge considering them also as users. Through these methodologies, we would integrate users from an initial stage and co-evaluate the status of the information design in the evacuation routes determined by local authorities, taking the problem designed from a macro scale to a detailed scale, making it possible for specific needs to appear. Due the pandemic, we were able to compare both systems, and design some visual improvements for a signage system that may be tested and evaluated on a second phase with both communities. 